\title{
ESTUDO LONGITUDINAL DO ELETROCARDIOGRAMA NA DOENÇA DE CHAGAS DESDE A FASE AGUDA
}

\author{
Roberto C. Pedrosa, J. Romeu Cançado e Waldemar Decache
}

\begin{abstract}
Vários trabalhos com eletrocardiograma na doença de Chagas têm sido feitos. Alguns referindo-se a grupos selecionados de casos, outros a estudos longitudinais, relatam as caracteristicas da mortalidade nas diversas fases da doença. Com o objetivo de avaliar o valor do eletrocardiograma como indice de avaliação terapêutica e de seu comportamento na doença de Chagas desde a fase aguda, no presente trabalho, analisou-se evolutivamente o eletrocardiograma de 42 pacientes (18 mulheres e 24 homens) procedentes da zona rural do Norte de Minıs Gerais; predominio elário foi nas duas primeiras décadas; todos com comprometimento cardiaco; todos receberam tratamento especifico. O acompanhamento dos 42 pacientes foi de 9 anos dos quais 3 pacientes tiveram seguimento de 20 anos. Foram analisados 270 eletrocardiogramas. Nós utilizamos os seguintes critérios para a análise do ECG: código de Minnesota modificado para doença de Chagas; WHO/I.S.F.C. TASK FORCE para condução intraventricular e critérios de Pierenti para área eletricamente inativa. Concluímos que as alterações eletrocardiográficas agravam com a evolução da doença e que o eletrocardiograma não serve de indice de avaliação terapêutica.
\end{abstract}

Palavras-chaves: Cardiopatia chagásica. Estudo longitudinal. Eletrocardiograma.

A doença de Chagas é endêmica no Continente Americano (América do Sul e Central) ${ }^{32} 41$ a tal ponto de que estimativas da Organização Mundial de Saúde apontam que entre 16 a 18 milhões de pessoas estão infectados com o Trypanosoma cruzi e que mais de 90 milhões são de risco 26314447 . No Brasil, recentemente, estimativas sorológicas mostrara 8 a 9 milhões de infectados dos quais aproximadamente 30 a $40 \%$ podem ter algum grau de comprometimento cardíaco sendo uma das principais causa de mortalidade na doença de Chagas $^{40}$.

A doença de Chagasé reconhecidamente doença polimórfica 1267891011121517192122232546 . Pode induzir distúrbios de condução no coração, incluindo arritmias ventriculares; em alguns casos, arritmias estão associadas à morte súbita ${ }^{3456910}$ 1516202735363750 .

Muitas controvérsias caracterizam teorias a

Serviço de Cardiologia do Hospital Universitário Clementino Fraga Filho da Faculdade de Medicina da Universidade Federal do Rio de Janeiro e Faculdade de Medicina da Universidade Federal de Minas Gerais.

Endereço para correspondência: Dr. Roberto Coury Pedrosa. Centro de Tratamento Intensivo do Hospital Universitário/ UFRJ. Av. Brig ${ }^{\circ}$ Trompowski $s / n^{\circ}, 13^{\circ}$ andar, Ilha do Fundão, 21941-680 Rio de Janeiro, RJ.

Recebido para publicação em 18/11/91. respeito de patogênese da cardiopatia chagásica crônica (CCC). Uma teoria atribui bases auto-imune ${ }^{29}$ 344254 enquanto outra implica no comprometimento primário do sistema nervoso autônomo levando a uma disfunção do mesmo ${ }^{33}$. Os mecanismos envolvidos no desenvolvimento das lesões cardíacas não são ainda completamente conhecidos, havendo várias possibilidades, entre as quais, a de que as lesões miocárdicas traduzam perturbações no sistema imunológico causadas pela interação entre o paciente e o parasita. Recentes estudos ${ }^{15} 243031455355$ apontam como provável mecanismo para explicar a patogênese da CCC, a lesão da microvasculatura do miocárdio resultando em áreas focais de hipoperfusão levando a uma progressiva disfunção ventricular devido a necrose miocelular, tecido fibrótico em substituição ao normal e hipertrofia reativa de células não afetadas. Isto tem encontrado apoio em estudos com eletrocardiograma, longitudinais ou estudos de grupos selecionados de casos, em que relatam as características da mortalidade nas diversas fases da doença.

Dias 2122 observou que alterações eletrocardiográficas consideradas graves na fase aguda se agravaram na fase crônica; alterações estas representadas por distúrbios de condução intraventriculares. Porto ${ }^{49}$ assinala que o encontro 
de hemibloqueio anterior esquerdo e as extrasístoles ventriculares associadas a bloqueio do ramo direito agravam o prognóstico, uma vez que foram cinco vezes mais freqüentes no seu grupo fatal. Maguire $^{40}$, em um estudo prospectivo de 1051 pacientes em área endêmica no Brasil, demonstrou uma progressiva incidência de anormalidades no eletrocardiograma principalmente bloqueio de ramo direito e hemibloqueio anterior esquerdo. Dias e cols $^{23}$ assinalam que a presença de bloqueio do ramo esquerdo indica prognóstico ruim, uma vez que a mortalidade em 5 e 10 anos após esses achados foi de $76 \%$ e $100 \%$ respectivamente. Caieiro e cols $^{12}$ estudaram a probabilidade de sobrevida de um grupo de 233 indivíduos com infecção pelo $T$. cruzi procedentes de Córdoba, Argentina observando que a sobrevida aos 10 anos de seguimento daqueles com alterações eletrocardiográficas e cardiomegalia foi de $78,2 \%$. Forichon ${ }^{25}$ analisou também as características da mortalidade em Bambuí entre indivíduos acompanhados no período de 1943 a 1973. Concluiu esse autor que na cardiopatia chagásica crônica o prognóstico é reservado, sobretudo nos indivíduos do sexo masculino e com alterações eletrocardiográficas. Coura e $\operatorname{cols}^{19} \mathrm{em}$ um estudo longitudinal de 10 anos (1974-1984) de um grupo de 235 indivíduos com infecção por $T$. cruzi observaram que a mortalidade por cardiopatia chagásica foi significativamente maior nos indivíduos do sexo masculino que no sexo feminino. Observou ainda que 67,5\% dos óbitos por cardiopatia chagásica ocorreram em indivíduos com alterações eletrocardiográficas.

É comum a todos os estudos longitudinais 1219 2125384046 que definiram grupo de risco de óbito, de acordo com as alterações previstas no início do seguimento, que a proporção de casos de óbito aumenta com a gravidade das alterações observadas no eletrocardiograma. Merece destaque especial a extra-sístole ventricular freqüente e multifocal, neste conjunto de alterações associado a um risco alto de óbito.

O presente trabalho buscará abordar a evolução da cardiopatia chagásica aguda e crônica, tendo como base $\mathbf{a}$ análise da evolução eletrocardiográfica desde a fase aguda com acompanhamento que em alguns casos chega a alcançar 20 anos e concomitantemente avaliar o valor do eletrocardiograma como índice de avaliação terapêutica.

\section{MATERIAL E MÉTODOS}

Foram estudados pacientes na fase aguda da doença de Chagas com acometimento do miocárdio, comprovado clínica, radiológica e eletrocardiograficamente. Os 45 pacientes eram provenientes da zona rural de 16 municípios do Norte de Minas Gerais e de 2 municípios do Sul da Bahia.

Os pacientes foram inicialmente internados no Hospital das Clínicas/UFMG, Belo Horizonte, durante a fase aguda, com finalidade de se submeterem a ensaios terapêticos (nifurtimox, benzonidazol) a nível de pesquisa, sempre dentro das normas de "ensaios terapêuticos clínicos controlados, obedecendo aos padrões éticos da Declaração de Helsinque". Após o mesmo, retornavam ao local de origem. $O$ acompanhamento a nivel ambulatorial foi feito na Fundação Carlos Chagas em Belo Horizonte por um mesmo médico (um dos autores). Durante o seguimento (19661975) perdemos $3(6,6 \%)$ pacientes.

Os critérios adotados para o diagnóstico da fase aguda da doença de Chagas foram os recomendados pelo Comitê da Organização Mundial de Saúde grupo Doença de Chagas, a saber: a) febre, às vezes febrículas; b) anemia e linfocitose relativa; c) residência em zona endêmica; d) pesquisa direta do T. cruzi no sangue periférico pelo exame a fresco e/ou xenodiagnóstico. Oacometimento do miocárdio observado deve-se à disfunção ventricular manifestada clinicamente na classe funcional I (oligossintomático) da New York Heart Association acompanhado de evidencias radiológicas principalmente índice cardiotorácico aumentado assim como, volume cardíaco aumentado.

Dos 302 ECG obtidos, foram analisados 270 , uma vez que $32(10,5 \%)$ foram abandonados por motivos vários (número insuficiente de derivações, má técnica, registros pequenos de ciclo, traçados ilegíveis). Tais ECGs se referem a tempos diferentes de evolução ao longo dos 9 anos. Assim tivemos 42 pacientes com seguimento de 9 anos dos quais 3 pacientes tiveram acompanhamento por 20 anos.

Para avaliar o valor do ECG como índice de avaliação terapêutica, os ECGs foram obtidos de forma convencional e diariamente desde o início da 
Pedrosa RC, Cançado JR, Decache W. Estudo longitudinal do eletrocardiograma na doença de Chagas desde a fase aguda. Revista da Sociedade Brasileira de Medicina Tropical 26:163-174, jul-set, 1993.

fase aguda até o desaparecimento da parasitemia (60-90 dias aposs). Até os primeiros 18 meses, o ECG foi repetido trimestralmente, depois de $6 \mathrm{em}$ 6 meses, o posteriormente, quando possível, de 2 em 2 anos a nível ambulatorial. Os ECGs iniciais foram colhidos antes que os pacientes tivessem iniciado o tratamento específico. Este grupo foi constituído de 27 dos 42 pacientes tratados.

Na interpretação do ECG utilizamos: a) código de Minnesota modificado para doença de Chagas ${ }^{39}$; b) os critérios estabelecidos pela WHO/I.S.F.C. TASK FORCE para os distúrbios na condução intraventricular; c) os critérios de Pierette da Sociedade Venezuelana de Cardiologia foram usados para interpretação de zona eletricamente inativa ${ }^{48}$.

Para verificar se haveria alterações eletrocardiográficas evolutivas diferentes em cada grupo na dependência de serem considerados curados ou não-curados, os pacientes foram separados em 2 grupos: a) grupo de pacientes curados; b) grupo de pacientes não-curados. Tivemos o cuidado de correlacionar a época de aparecimento da anormalidade com a persistência de cada uma das alterações registradas no ECG. Definimos como critério de cura a negatividade tanto das reações sorológicas como de xenodiagnóstico. Para uma melhor análise evolutiva dos eletrocardiogramas durante os 9 anos de seguimento (1966-1975) os pacientes foram separados em 2 grupos: grupo I ECGs normais durante a fase aguda; grupo II ECGs alterados durante a fase aguda.

\section{RESULTADOS}

Dos 42 pacientes chagásicos, 18 eram do sexo feminino e 24 do sexo masculino, cuja distribuição etária adquirida via vetor 6 a seguinte: a) $35,2 \%$ de 0-10 anos; b) $32,4 \%$ de 11-20 anos; c) $13,5 \%$ de 21 30 anos; d) 8,1\% de 31-40 anos e 5,4 de 41-60 anos.

A observação da idade do paciente (Figura 1 e Tabela 1) ao contrair a infecção, por via do vetor natural, confirma a preponderância nas duas primeiras décadas de vida, mas permite também verificar a ocorrência em qualquer idade.

Os pacientes provinham quase todos de área endêmica do Norte de Minas Gerais; oito (20\%) deles se infectaram por via transfusão sanguínea; em 11 dos 37(30\%), que se infectaram naturalmente, não se viam os sinais de porta de entrada (sinal de Romaña-complexo oftalmoganglionar- e os chagomias de inoculação); o exame usado para comprovar o diagnóstico foi o direto do sangue periférico, a fresco, que revelou o parasito nos $40(89 \%)$ pacientes em plena fase aguda e nos $5(11 \%)$ pacientes restantes pelo xenodiagnóstico (Tabela 1).

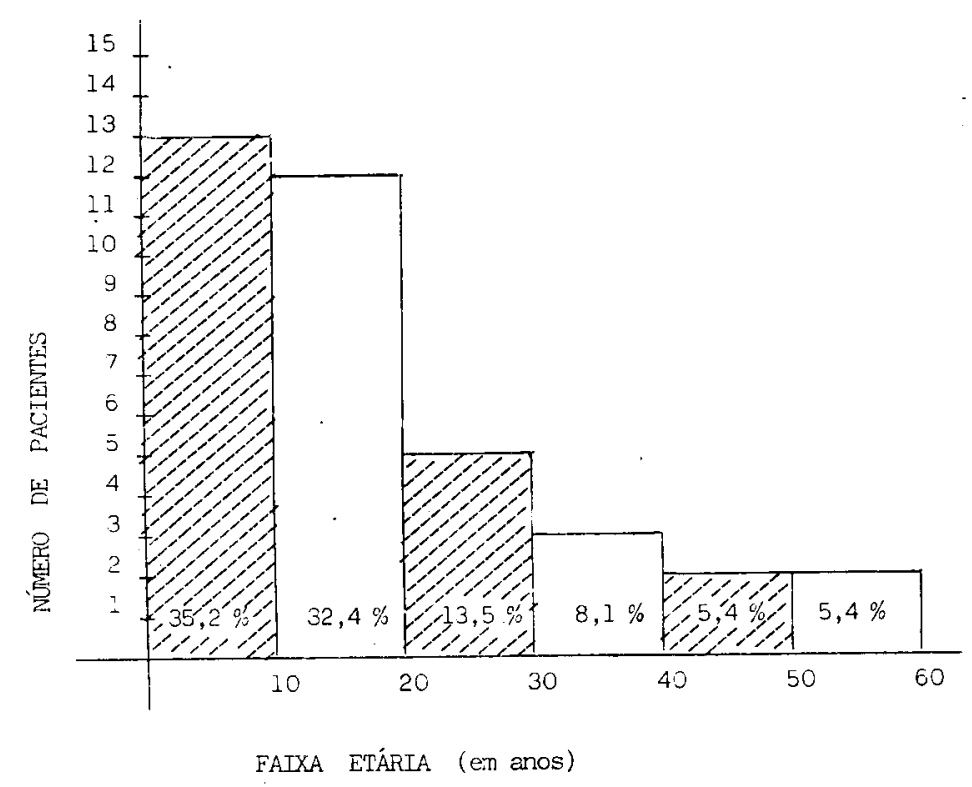

Figura 1 - Distribuição etária de 37 pacientes da doença de Chagas aguda, adquirida via vetor. 
Pedrosa RC, Cançado JR, Decache W. Estudo longitudinal do eletrocardiograma na doença de Chagas desde a fase aguda. Revista da Sociedade Brasileira de Medicina Tropical 26:163-174, jul-set, 1993.

Tabela 1 - Casos de doença de Chagas aguda diagnosticada durante os anos de 1966 - 1968.

\begin{tabular}{|c|c|c|c|c|c|c|c|c|}
\hline \multirow[t]{2}{*}{ Caso } & \multirow[t]{2}{*}{ Nome } & \multirow[t]{2}{*}{ Idade } & \multirow[t]{2}{*}{ Procedência } & \multirow[t]{2}{*}{ Fase aguda } & \multicolumn{2}{|c|}{ Transmissão } & \multicolumn{2}{|c|}{ Eletrocardiograma } \\
\hline & & & & & & & fase aguda & após 9 anos \\
\hline 1 & JLO & 0,4 & B. Horizonte & 27.05 .66 & & ET & $A R V+B V+T S$ & HBAE + BRD \\
\hline 2 & MIS & 12,0 & Bocaíuva & 20.06 .66 & $\mathbf{N}$ & & $\mathrm{ARV}+\mathrm{BV}$ & $\mathbf{N}$ \\
\hline 3 & LLS & 24,0 & Ubaí & 16.07 .67 & $\mathbf{N}$ & & BV & RV \\
\hline 4 & CRS & 5,0 & Espinosa & 24.02 .72 & $\mathbf{N}$ & & - & $\mathrm{N}$ \\
\hline 5 & GFSA & 33,0 & M. Claros & 01.06 .73 & $\mathrm{~N}$ & & ARV & $\mathbf{N}$ \\
\hline 6 & WRJ & 18,0 & Franc. Sá & 15.11 .73 & $\mathbf{N}$ & & $\mathrm{ARV}+\mathrm{TS}$ & HBAE \\
\hline 7 & AAS & 18,0 & Franc. Sá & 15.01 .74 & $\mathbf{N}$ & & $\mathrm{HBPE}+\mathrm{TS}$ & HBPE \\
\hline 8 & MAB & 39,0 & Unaí & 19.03 .74 & $\mathbf{N}$ & & $\mathrm{BV}+\mathrm{TS}$ & $\mathbf{N}$ \\
\hline $9 *$ & GXS & 9,0 & B. Horizonte & 10.04 .74 & & $\mathrm{~T}$ & - & - \\
\hline $10 * *$ & MLC & 48,0 & M. Claros & 05.01 .74 & & $\mathrm{~T}$ & ARV & (óbito) \\
\hline 11 & GMS & 14,0 & M. Claros & 05.07 .74 & & $\mathrm{~T}$ & $\mathrm{FA}+\mathrm{BV}+\mathrm{BRD}$ & M. Súbita \\
\hline 12 & VLSC & 7,0 & Franc. Sá & 25.08 .74 & $\mathrm{~N}$ & & HBPE & HBPE \\
\hline 13 & CMC & 16,0 & Jaiba & 01.09 .74 & $\mathbf{N}$ & & BV & $\mathrm{N}$ \\
\hline 14 & JMA & 6,0 & Franc. Sá & & $\mathbf{N}$ & & $\mathrm{ARV}+\mathrm{BV}$ & $\mathrm{N}$ \\
\hline 15 & JBS & 0,7 & M. Claros & 29.09 .74 & & $\mathrm{~T}$ & $\mathbf{N}$ & $\mathrm{N}$ \\
\hline 16 & MSFS & 34,0 & Franc. Sá & 16.10 .74 & $\mathbf{N}$ & & ARV & $\mathbf{N}$ \\
\hline 17 & DMF & 14,0 & Franc. Sá & 20.10 .74 & $\mathrm{~N}$ & & TS & $\mathbf{N}$ \\
\hline 18 & MAJ & 15,0 & Franc. Sá & 10.11 .74 & $\mathrm{~N}$ & & - & $\mathrm{N}$ \\
\hline 19 & JFS & 20,0 & Franc. Sá & 25.12 .74 & $\mathbf{N}$ & & $\mathrm{N}$ & BRD \\
\hline 20 & JNO & 1,0 & M. Claros & 20.03 .75 & $\mathbf{N}$ & & $\mathbf{N}$ & $\mathbf{N}$ \\
\hline 21 & MLR & 42,0 & Franc. Sá & 28.05 .75 & $\mathbf{N}$ & & TS & $N$ \\
\hline 22 & GBS & 5,0 & M. Claros & 25.06 .75 & $\mathbf{N}$ & & TS & $\mathbf{N}$ \\
\hline 23 & JSS & 46,0 & M. Claros & 08.11 .75 & $\mathbf{N}$ & & $\mathrm{N}$ & $\mathbf{N}$ \\
\hline 24 & JES & 6,0 & Franc. Sá & 14.12 .75 & $\mathbf{N}$ & & ARV & $\mathrm{N}$ \\
\hline 25 & RRA & 2,0 & Brasilândia & 20.01 .76 & $\mathbf{N}$ & & $A R V+B R D$ & $\mathrm{BRD}+\mathrm{ARV}$ \\
\hline 26 & LAS & 3,0 & Jaibena & 29.03 .76 & $\mathbf{N}$ & & $\mathrm{HBAE}+\mathrm{ARV}$ & $\mathrm{HBAE}+\mathrm{ARV}$ \\
\hline 27 & IFR & 29,0 & Unaí & 20.08 .76 & $\mathrm{~N}$ & & - & $N$ \\
\hline 28 & ARB & 14,0 & M. Claros & 06.09 .76 & $\mathbf{N}$ & & ARV & ARV \\
\hline 29 & BFF - & 38,0 & Ubaí & 15.09 .76 & $\mathbf{N}$ & & $\mathbf{N}$ & $\mathbf{N}$ \\
\hline 30 & SCPL & 13,0 & M. Claros & 20.01 .77 & $\mathbf{N}$ & & $\mathbf{N}$ & $\mathrm{N}$ \\
\hline 31 & LFT & 13,0 & Cuanambi & 05.02 .77 & $\mathbf{N}$ & & HBAE + ARV & $\mathrm{HBAE}+\mathrm{ARV}$ \\
\hline 32 & CNTN & 7,0 & Montalcânia & 23.07 .77 & $\mathbf{N}$ & & $\mathbf{N}$ & $N$ \\
\hline 33 & CMP & 23,0 & Espinosa & 05.08 .77 & $\mathrm{~N}$ & & $\mathbf{N}$ & $\mathbf{N}$ \\
\hline 34 & BRS & 5,0 & M. Claros & 05.08 .77 & $\mathbf{N}$ & & $\mathrm{TS}+\mathrm{BV}$ & $\mathbf{N}$ \\
\hline 35 & EFS & 25,0 & Januária & 25.08 .77 & $\mathbf{N}$ & & ARV & ARV \\
\hline 36 & JMB & 27,0 & Janaiba & 10.10 .77 & $\mathbf{N}$ & & TS & $\mathbf{N}$ \\
\hline 37 & $\mathrm{COC}$ & 14,0 & Espinosa & 01.12 .78 & $\mathbf{N}$ & & $\mathbf{N}$ & $\mathbf{N}$ \\
\hline 38 & NRT & 9,0 & M. Azul & 25.02 .78 & $\mathbf{N}$ & & $\mathbf{N}$ & $\mathbf{N}$ \\
\hline 39 & OP & 0,5 & En. Dolab. & 01.05 .78 & $\mathbf{N}$ & & $\mathrm{ARV}+\mathrm{TS}$ & $\mathrm{ARV}+\mathrm{TS}$ \\
\hline 40 & MVC & 60,0 & Jaiba & 08.06 .78 & $\mathbf{N}$ & & $\mathbf{N}$ & $\mathbf{N}$ \\
\hline 41 & EBS & 9,0 & Barreiros & 30.09 .78 & $\mathbf{N}$ & & $\mathrm{N}$ & $\mathbf{N}$ \\
\hline 42 & JGVS & 11,0 & Grão Mogol & 26.02 .79 & $\mathbf{N}$ & & $A R V+B V$ & $\mathbf{N}$ \\
\hline 43 & WBF & 27,0 & Acesita & 28.02 .79 & & $\mathrm{~T}$ & $\mathbf{N}$ & $\mathbf{N}$ \\
\hline 44 & TAS & 58,0 & Grão Mogol & 10.03 .79 & $\mathbf{N}$ & & BV & $\mathbf{N}$ \\
\hline 45 & ZKS & 35,0 & Curitiba & 24.09 .79 & & $\mathrm{~T}$ & - & $\mathbf{N}$ \\
\hline
\end{tabular}

$\mathrm{N}=$ natural; $\mathbf{T}=$ transfusão; $\mathbf{E T}=$ ex. sanguíneo; * leucose; ** óbito de CA mama.

Diagnóstico confirmado por exame a fresco (40 casos) salvo por xenodiagnóstico ( $\left.n^{\circ} 21,30,32,36,43\right)$.

Eletrocardiografia: explicação das abreviaturas. 
O índice cardiotorácico (ICT), que é um dado objetivo, de avaliação do tamanho cardíaco, revelouse normal em $53 \%$ e aumentado em $47 \%$, de grau leve ou moderado. Observa-se preponderância de aumento do ICT na primeira década na relação de 10:3 (ver Tabela 7).

A análise dos traçados eletrocardiográficos do grupo de pacientes curados (grupo a - ver Tabela 2): quatorze pacientes tinham ECG seguidos por 9 anos, idade variando de 0,7 a 60 anos. $\mathrm{Na}$ fase aguda, os ECGs iniciais, isto é, de pacientes ainda não-tratados, comparados com os traçados após 6090 dias mantiveram as mesmas alterações em $93 \%$ dos mesmos. Distúrbios de condução intraventricular manifestado por hemibloqueio anterior esquerdo ocorreram em 3 pacientes ( ${ }^{\circ}$. 6,26 e 31 ), sendo que, em 2 deles ( $n^{\circ} \cdot 26$ e 31 ), persistiram ao longo dos 9 anos, e em 1 paciente ( $\left.\mathrm{n}^{\circ} \cdot 6\right)$ foi se manifestar após 12 anos de seguimento. Setenta e dois por cento dos pacientes tiveram seus traçados eletrocardiográficos normais desde a fase aguda até os 9 anos de seguimento.

No grupo de pacientes não-curados (grupo b Tabela 3) temos 13 pacientes dos quais $12 \mathrm{com}$ seguimento eletrocardiográfico por 9 anos, idade variando de 0,4 a 58 anos. Na fase aguda 4 pacientes não mantiveram as mesmas alterações eletrocardiográficas após 60-90 dias. Houve 4 pacientes que tinham o ECG alterado antes do tratamento e que normalizaram após 60-90 dias. Houve 2 pacientes (no. 7 e 12) com hemibloqueio posterior esquerdo na fase aguda antes do tratamento, que persistiu ao longo dos 9 anos. Houve 1 paciente $\left(\mathrm{n}^{\mathrm{o}} \cdot 25\right)$ durante a fase aguda apresentou bloqueio de ramo direito eque ao longo do seguimento, progrediu para sua forma completa; 1 paciente $\left(n^{\circ} \cdot 1\right)$ apresentou hemibloqueio anterior esquerdo no $5^{\circ}$ ano de observação e bloqueio de ramo direito no $13^{\circ}$ ano alterações essas que persistiram até o $20^{\circ}$ ano.

Na Tabela 4 observamos a evolução eletrocardiográfica de distúrbios de condução intraventricular, constituída de 9 pacientes com idade variando de 0,4 a 20 anos, isto é, a maioria enquadra-se no grupo de baixa idade. Novamente aqui observamos que o tratamento específico não ocasionou nenhuma modificação eletrocardiográfica evolutiva. O hemibloqueio anterior esquerdo antecedendo o bloqueio de ramo direito. Progressão quanto ao grau de bloqueio de ramo direito também

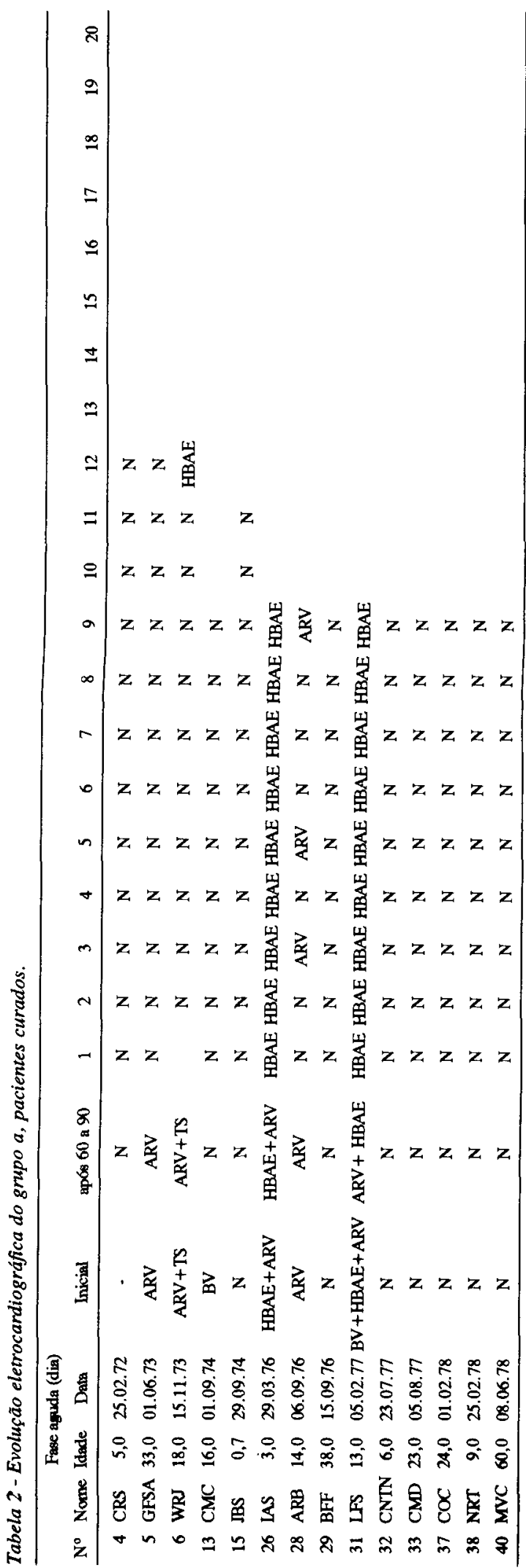




\begin{tabular}{|c|c|c|c|c|c|c|c|c|c|c|c|c|c|c|c|c|c|c|c|c|c|c|c|c|c|}
\hline \multirow[b]{2}{*}{$\mathrm{N}^{\circ}$} & \multicolumn{4}{|c|}{ Fase aguda (dia) } & \multirow[b]{2}{*}{ aṕs 60 a 90} & \multirow[b]{2}{*}{1} & \multirow[b]{2}{*}{2} & \multirow[b]{2}{*}{3} & \multirow[b]{2}{*}{4} & \multirow[b]{2}{*}{5} & \multirow[b]{2}{*}{6} & \multirow[b]{2}{*}{7} & \multirow[b]{2}{*}{8} & \multirow[b]{2}{*}{9} & \multirow[b]{2}{*}{10} & \multirow[b]{2}{*}{11} & \multirow[b]{2}{*}{12} & \multirow[b]{2}{*}{13} & \multirow[b]{2}{*}{14} & \multirow[b]{2}{*}{15} & \multirow[b]{2}{*}{16} & \multirow[b]{2}{*}{17} & \multirow[b]{2}{*}{18} & \multirow[b]{2}{*}{19} & \multirow[b]{2}{*}{20} \\
\hline & Nome & Idede & Data & Inicial & & & & & & & & & & & & & & & & & & & & & \\
\hline 1 & FLO & 0,4 & 27.05 .66 & $A R V+B V+T S$ & $\mathrm{ARV}+\mathrm{BV}+\mathrm{TS}$ & ARV & ARV & ARV & ARV & HBAE & $E$ HBAE & HBAE & HBAE & 5 HAAE & HAAE & HBAE & HBAF & HBAE & HBAE & HBAE & HBAE & HBAE & HBAE & HBAE & HBAE \\
\hline & & & & & & TS & BV & BV & BV & & BV & & & & BV & & & $\begin{array}{c}\text { BRD } \\
\text { IIG }\end{array}$ & $\begin{array}{c}\text { BRD } \\
\text { IIG }\end{array}$ & $\begin{array}{l}\text { BRD } \\
\text { IIG }\end{array}$ & $\begin{array}{c}\text { BRD } \\
\text { IIG }\end{array}$ & BRD & $\mathrm{BRD}$ & BRD & BRD \\
\hline 2 & MIS & 12,0 & 20.06 .66 & $A R V+B V$ & $\mathrm{ARV}+\mathrm{BV}$ & $\mathrm{N}$ & $\mathbf{N}$ & $\mathbf{N}$ & $\mathbf{N}$ & $\mathbf{N}$ & $\mathbf{N}$ & $\mathbf{N}$ & $\mathbf{N}$ & $\mathbf{N}$ & $\mathbf{N}$ & $\mathbf{N}$ & $\mathbf{N}$ & $\mathbf{N}$ & ARV & ARV & ARV & ARV & ARV & ARV & ARV \\
\hline 3 & LLS & 24,0 & 16.07 .67 & BV & BV & $\mathbf{N}$ & $N$ & $\mathbf{N}$ & $\mathbf{N}$ & $\mathbf{N}$ & $\mathbf{N}$ & BV & BV & BV & BV & BV & BV & BV & BV & BV & BV & BV & BV & $\mathrm{BV}$ & $\mathrm{BV}$ \\
\hline 7 & AAS & 18,0 & 15.01 .74 & HBPE + TS + BV & $\mathrm{HBPE}+\mathrm{TS}+\mathrm{BV}$ & HBPE & HBPE & HBPE & HBPE & HBPE & HBPE & HBPE & HBPE & HBPE & HBPE & HBPE & & & & & & & & & \\
\hline 12 & VLSC & 7,0 & 25.08 .74 & $\mathrm{ARV}+\mathrm{HBP}$ & HBPE & HBPE & HBPE & HBPE & HBPE & HBPE & HBPE & HBPE & HBPE & HBPE & HBPE & & & & & & & & & & \\
\hline 17 & DMS & 14,0 & 20.10 .74 & TS & N & $\mathbf{N}$ & $\mathbf{N}$ & $\mathbf{N}$ & $\mathrm{N}$ & $\mathbf{N}$ & $\mathbf{N}$ & $\mathbf{N}$ & $\mathbf{N}$ & $\mathbf{N}$ & $\mathbf{N}$ & $\mathbf{N}$ & & & & & & & & & \\
\hline 24 & JES & 6,0 & 14.02 .75 & ARV & $\mathbf{N}$ & $\mathbf{N}$ & $\mathbf{N}$ & $\mathbf{N}$ & $\mathbf{N}$ & $\mathbf{N}$ & $\mathbf{N}$ & $\mathbf{N}$ & $\mathbf{N}$ & $\mathbf{N}$ & $\mathbf{N}$ & & & & & & & & & & \\
\hline 25 & RAA & 2,0 & 20.01 .76 & $A R V+B R D$ & $A R V+B R D$ & BRD & BRD & $\mathrm{BRD}$ & BRD & BRD & BRD & BRD & BRD & BRD & & & & & & & & & & & \\
\hline & & & & $1^{\circ} \mathrm{G}$ & $2^{\circ} \mathrm{G}$ & $2^{\circ} \mathrm{G}$ & $2^{\circ} \mathrm{G}$ & $3^{\circ} \mathrm{G}$ & $3^{\circ} \mathrm{G}$ & $3^{\circ} \mathrm{G}$ & $3^{\circ} \mathrm{G}$ & $3^{\circ} \mathrm{G}$ & $3^{\circ} \mathrm{G}$ & $3^{\circ} \mathrm{G}$ & & & & & & & & & & & \\
\hline 27 & IFR & 29,0 & 20.08 .76 & - & $\mathbf{N}$ & $\mathbf{N}$ & $\mathbf{N}$ & $\mathbf{N}$ & $\mathbf{N}$ & $\mathrm{N}$ & $\mathbf{N}$ & $\mathbf{N}$ & $\mathbf{N}$ & $\mathbf{N}$ & $\mathbf{N}$ & & & & & & & & & & \\
\hline 35 & EFS & 25,0 & 25.08 .76 & $\mathrm{ARV}$ & ARV & ARV & ARV & ARV & ARV & ARV & $\mathbf{N}$ & $\mathbf{N}$ & $\mathbf{N}$ & ARV & & & & & & & & & & & \\
\hline 39 & $J P$ & 0,5 & 01.05 .78 & $\mathrm{TS}+\mathrm{ARV}$ & $\mathrm{TS}+\mathrm{ARV}$ & - & Ext.V & & & & & & & & & & & & & & & & & & \\
\hline 42 & JGVS & 11,0 & 26.02 .77 & $\mathrm{ARV}+\mathrm{BV}$ & $\mathbf{N}$ & $\mathbf{N}$ & $\mathbf{N}$ & $\mathrm{N}$ & $\mathbf{N}$ & $\mathbf{N}$ & $\mathbf{N}$ & $\mathbf{N}$ & $\mathbf{N}$ & $\mathbf{N}$ & & & & & & & & & & & \\
\hline 44 & TAS & 58,0 & 10.03 .77 & BV & $\mathbf{N}$ & $\mathbf{N}$ & $\mathrm{N}$ & $\mathrm{N}$ & $\mathrm{N}$ & $\mathbf{N}$ & $\mathbf{N}$ & $\mathrm{N}$ & $\mathbf{N}$ & $\mathbf{N}$ & & & & & & & & & & & \\
\hline
\end{tabular}

Tabela 4 - Evolução eletrocardiográfica de distúrbios de condução intraventricular de 42 casos de doença de Chagas.

\begin{tabular}{|c|c|c|c|c|c|c|c|c|c|c|c|c|c|c|c|c|c|c|c|c|c|c|c|c|c|}
\hline \multirow[b]{2}{*}{$\mathbf{N}^{\circ}$} & \multicolumn{4}{|c|}{ Fase aguda (dia) } & \multirow[b]{2}{*}{ após 60 a 90} & \multicolumn{20}{|c|}{ Fase cronica (mno) } \\
\hline & Nome & Idade & Data & Inicial & & 1 & 2 & 3 & 4 & 5 & 6 & 7 & 8 & 9 & 10 & 11 & 12 & 13 & 14 & 15 & 16 & 17 & 18 & 19 & 20 \\
\hline \multirow[t]{3}{*}{1} & ภLO & 0,4 & 27.05 .66 & $\mathrm{ARV}+\mathrm{BV}+\mathrm{TS}$ & $\mathrm{ARV}+\mathrm{BV}+\mathrm{TS}$ & ARV & ARV & ARV & ARV & ARV & ARV & ARV & ARV & ARV & HBAE & HBAE & HBAE & HRAE & HBAE & HBAF & HBAE & HRAE & HBAE & HBAE & HAAE \\
\hline & & & & & & $\mathrm{TS}$ & BV & BV & BV & & BV & & & & & & & BRD & BRD & & BRD & $\mathrm{BRD}$ & BRD & BRD & BRD \\
\hline & & & & & & & & & & & & & & & & & & IIG & IIG & & IIIG & & & & \\
\hline 6 & WRJ & 18,0 & 15.11 .73 & $\mathrm{ARV}+\mathrm{TS}$ & $\mathrm{ARV}+\mathrm{TS}$ & $\mathbf{N}$ & $\mathbf{N}$ & $\mathbf{N}$ & $\mathrm{N}$ & $\mathrm{N}$ & $N$ & $\mathbf{N}$ & $\mathbf{N}$ & $\mathbf{N}$ & $\mathbf{N}$ & $\mathbf{N}$ & HBAE & & & & & & & & \\
\hline 7 & AAS & 18,0 & 15.01 .74 & HBPE + TS + BV & HBPE+TS & HBPE & HBPE & HBPE & HBPE & HBPE & HBPE & HBPE & HBPE & HBPE & HBPE & HBPE & & & & & & & & & \\
\hline 11 & GMS & 14,0 & 05.07 .74 & $\mathrm{FA}+\mathrm{BV}+\mathrm{BAV}+$ & & & & & & & & & & & & & & & & & & & & & \\
\hline & & & & $\mathrm{TS}+\mathrm{BRD} 1^{\circ} \mathrm{grau}$ & & & & & & & & & & & & & & & & & & & & & \\
\hline 19 & JFS & 20.0 & 25.12 .74 & $\mathbf{N}$ & $\mathbf{N}$ & $\mathbf{N}$ & $\mathbf{N}$ & $\mathbf{N}$ & $\mathbf{N}$ & - & - & - & - & BRD & & & & & & & & & & & \\
\hline 25 & RAA & 2,0 & 20.01 .76 & $A R V+B R D$ & $A R V+B R D$ & BRD & BRD & BRD & BRD & $\mathrm{BRD}$ & BRD & BRD & BRD & BRD & & & & & & & & & & & \\
\hline & & & & $1^{\circ} \mathrm{grau}$ & $2^{\circ} \mathrm{grau}$ & $2^{\circ} \mathrm{G}$ & $2^{\circ} \mathrm{G}$ & $3^{\circ} \mathrm{G}$ & $3^{\circ} \mathrm{G}$ & $3^{\circ} \mathrm{G}$ & $3^{\circ} \mathrm{G}$ & $3^{\circ} \mathrm{G}$ & $3^{\circ} \mathrm{G}$ & $3^{\circ} \mathrm{G}$ & & & & & & & & & & & \\
\hline 26 & IAS & 3,0 & 29.03 .76 & HBAE + ARV & HBAE+ARV & HBAE & HBAE & HBAE & HBAE & HBAE & HBAE & HBAE & HBAE & HBAE & & & & & & & & & & & \\
\hline 31 & LFS & 13,0 & 05.02 .77 & $\begin{array}{c}\mathrm{BV}+\mathrm{HBAE}+ \\
\mathrm{ARV}\end{array}$ & $A R V+H B A E$ & HBAE & HBAE & HBAE & HBAE & HBAE & HBAE & HBAE & HBAE & HRAE & & & & & & & & & & & \\
\hline 42 & VISC & 7,0 & 25.08 .74 & $\mathrm{ARV}+\mathrm{HBPE}$ & HBPE & HBPE & HBPE & HBPE & HBPE & HBPE & HBPE & HBPE & HBPE & HBPE & & & & & & & & & & & \\
\hline
\end{tabular}


Pedrosa RC, Cançado JR, Decache W. Estudo longitudinal do eletrocardiograma na doença de Chagas desde a fase aguda. Revista da Sociedade Brasileira de Medicina Tropical 26:163-174, jul-set, 1993.

foi observado. Nota-se permanência dos distúrbios de condução intraventricular durante a fase crônica.

$\mathrm{Na}$ Tabela 5 que mostra a evolução eletrocardiográfica da fase aguda, antes e após tratamento dos 27 pacientes, notamos que praticamente não houve modificações eletrocardiográficas evolutivas diferentes. As principais alterações em ordem de freqüência foi a taquicardia sinusal, alterações de repolarização ventricular e a baixa voltagem. Fato relevante foi a presença de 2 casos de hemibloqueio posterior esquerdo não relatados na literatura (Figura 2).

\begin{tabular}{|c|c|c|c|c|}
\hline \multirow{2}{*}{ Diagnóstico* } & \multicolumn{2}{|c|}{ Inicial } & \multicolumn{2}{|c|}{$\begin{array}{l}\text { Eletrocardiograma } \\
\text { após } 60 \text { - } 90 \text { dias }\end{array}$} \\
\hline & $n^{0}$ & $\%$ & $n^{0}$ & $\%$ \\
\hline Normal & 11 & 30,5 & 15 & 41,6 \\
\hline Taquicardia sinusal & 8 & 22,2 & 8 & 22,2 \\
\hline Baixa voltagem de $Q R S$ & 10 & 27,7 & 6 & 16,6 \\
\hline Alteração ST & 7 & 19,4 & 3 & 8,3 \\
\hline $\mathbf{T}$ & 10 & 27,7 & 7 & 19,4 \\
\hline HBAE & 2 & 5,5 & 2 & 5,5 \\
\hline HBPE & 2 & 5,5 & 2 & 5,5 \\
\hline BAV $1^{\circ} \mathrm{grau}$ & 1 & 2,7 & 1 & 2,7 \\
\hline Fibrilação atrial & 1 & 2,7 & 1 & 2,7 \\
\hline BRD & 2 & 5,5 & 1 & 2,7 \\
\hline
\end{tabular}

* Duas ou mais alterações podem estar associadas no mesmo traçado excluídos os normais.

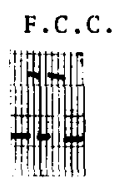

$\mathrm{mV}$

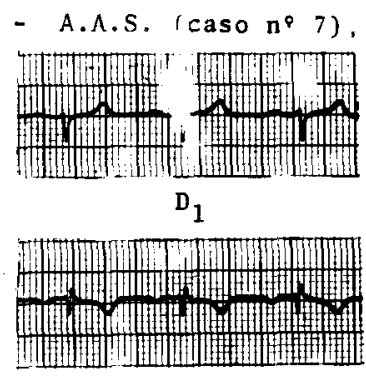

aVR
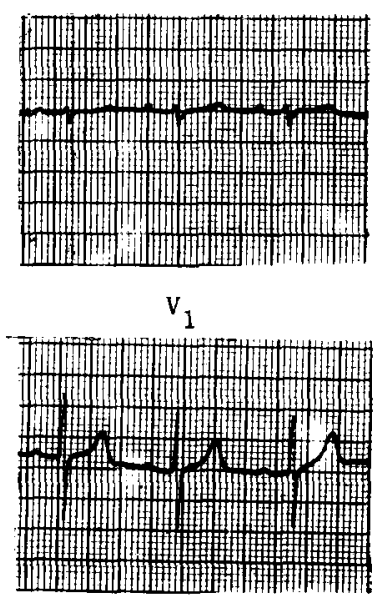

$\mathrm{v}_{4}$

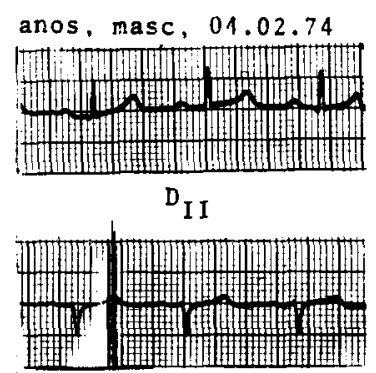

aVL

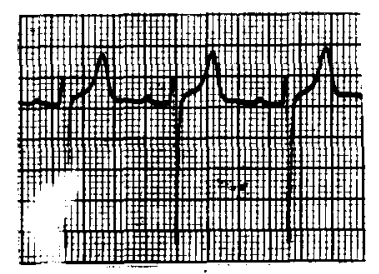

$\mathrm{v}_{2}$

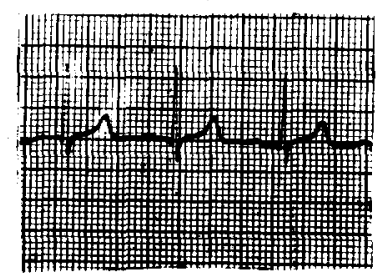

$v_{5}$

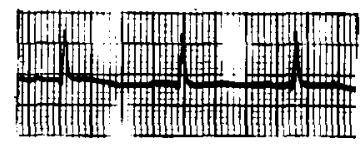

D I I I $_{\text {II }}$

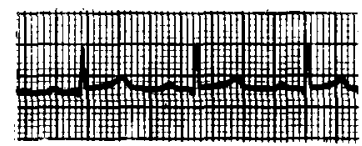

aVF

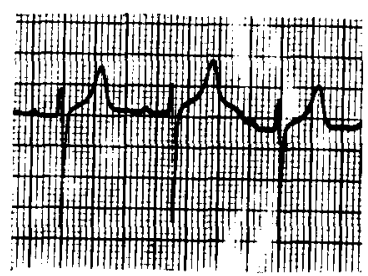

$\mathrm{v}_{3}$

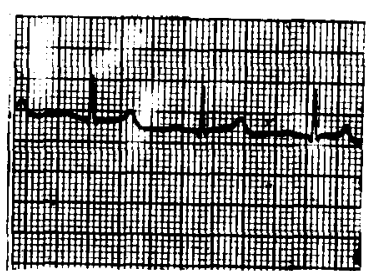

$v_{6}$

Figura 2 - Hemibloqueio posterior esquerdo (fase aguda). 
$\mathrm{Na}$ Tabela 6 apresentamos uma análise dos ECGs dös 42 pacientes chagásicos em que $30,5 \%$ dos mesmos na fase aguda foram normais e $69,5 \%$ anormais. Evidenciamos que, à medida que progridemos anos de acompanhamento, observamos um menor número de ECGs normais. $\mathrm{Na}$ análise eletrocardiográfica com intervalo de 5 anos, podemos verificar na tabela em apreço que houve uma incidência crescente de novas alterações, na proporção de 2,52\% ao ano.

Na Figura 3 observamos que no grupo I não houve variações evolutivas eletrocardiográficas durante os 9 anos de seguimento. No grupo II houve uma regressão acentuada das modificações eletrocardiográficas no primeiro ano após a fase aguda. Entre o oitavo e o nono ano de seguimento nota-se um aumento do número de pacientes com o ECG alterado.

\section{DISCUSSÃO}

\section{Eletrocardiograma na cardiopatia chagásica aguda}

O quadro evolutivo do ECG na fase aguda foi descrito nos trabalhos clássicos de Laranja e cols ${ }^{23}$ 35 36. Em nosso material o ECG apresentou-se normal em 30,5\% dos casos e, ao fim dos 3 primeiros anos de infecção, a normalidade eletrocardiográfica subiu para $62 \%$. Na casuística de Bambui ${ }^{12}$ com 313 casos, foi observado comportamento semelhante, pois $63,3 \%$ dos pacientes mostravam ECG normal na fase aguda, alcançando $75 \%$ de normalidadeno final do primeiro ano. Na literatura verifica-se o bom prognóstico imediato (cinco a dez anos) dos chagásicos com ECGs normais 78910212235364951 .

Tabela 6 - Análise dos eletrocardiogramas dos 42 casos de doença de Chagas.

\begin{tabular}{|c|c|c|c|c|c|}
\hline & \multicolumn{2}{|c|}{ Fase aguda } & & \multicolumn{2}{|c|}{ Fase crônica } \\
\hline & $\mathrm{n}^{\mathbf{0}}$ & $\%$ & & $n^{0}$ & $\%$ \\
\hline total & 42 & 100,0 & total & 42 & 100,0 \\
\hline normais & 13 & 30,5 & normais até 5 anos & 16 & 37,8 \\
\hline \multirow[t]{3}{*}{ anormais } & 29 & 69,5 & normais 5 a 10 anos & 15 & 35,6 \\
\hline & & & normais 10 a 15 anos & 4 & 11,1 \\
\hline & & & normais 15 a 20 anos & 0 & 0,0 \\
\hline
\end{tabular}

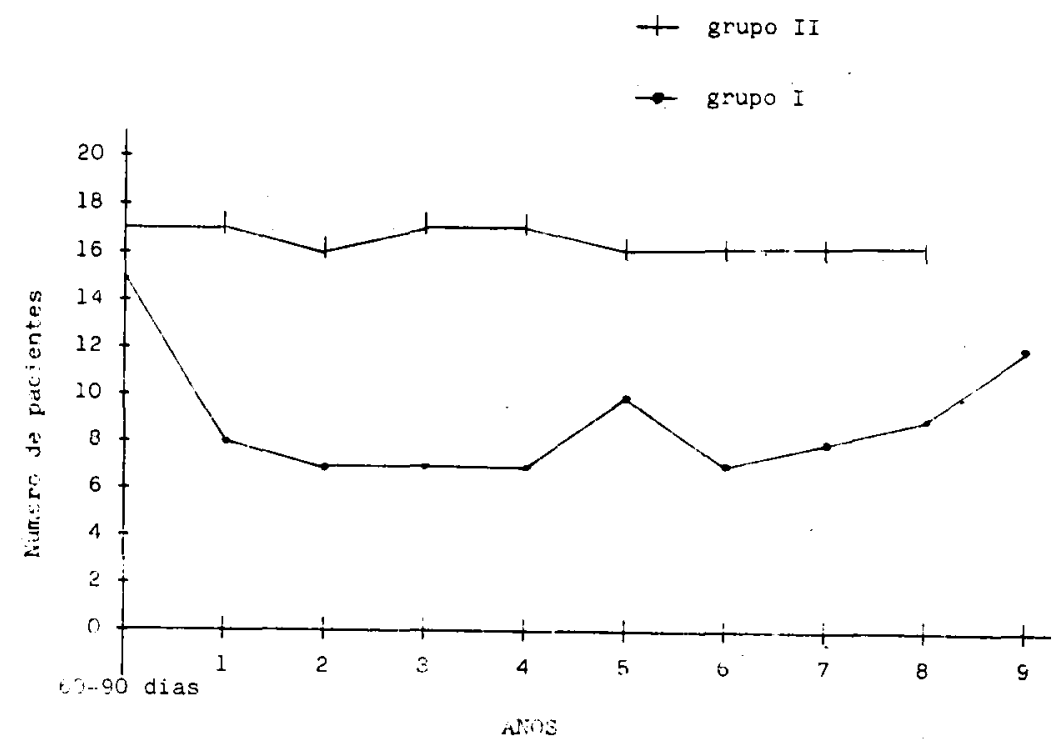

Figura 3 - Evolução eletrocardiográfica da fase crônica da doença de Chagas. 
Relata-se também que nos primeiros anos após a fase aguda podem regredir as modificações eletrocardiográficas, permanecendo os pacientes por muitos anos com ECG normal. Tal é explicado pela natureza inflamatória do processo na fase aguda da doença 22234 .

Assinalamos a presença de distúrbios de condução nos sub-ramos do feixe esquerdo. Isto é, HBAE e HBPE, registrados ambos em 5,5\% de nosso material, sendo este último incomum em outros relatos. Segundo alguns autores 2122 tais alterações podem permanecer por longos anos sem evolução aparente, seriam elas dependentes de processos inflamatórios não muito éxtensos e em atividade (miocardite fibrosante crônica). Outros entretanto enfatizam a noção de maior gravidade destes distúrbios de conduçāo que seriam tanto mais indicativos de pior prognóstico quanto mais jovem fosse o paciente infectado.

A taquicardia sinusal esteve presente em nosso material em 22,2\% na fase aguda (Tabela 5). Chagas ${ }^{17}$ mencionou a existência da taquicardia, em geral independente de febre e de hipotensão arterial, atribuindo ao ataque do miocárdio pelo $T$. cruzi. Ela esteve presente em $85 \%$ dos 31 casosagudos diagnosticados em Bambui ${ }^{21}$, muitas vezes não guardando nenhuma relação com o grau de elevação térmica. Na opinião de Körbele ${ }^{33}$, a taquicardia somente resultaria da destruição parassimpática do coração com consequente predominância relativa do simpático.

Em nosso material, baixa voltagem ocorreu em $27,7 \%$ no início da fase aguda e após $60-90$ dias em $16,6 \%$ (Tabela 5) comportamento esse já descrito por diferentes autores. Acredita-se que ela representaria a diminuição da atividade elétrica do miocárdio em virtude do envolvimento difuso pelo processo inflamatório.

As conseqüências a longo prazo (fase crônica) da cardiopatia chagásica aguda dependeriam da intensidade do processo inflamatório, da desnervação ou de ambos os fatores na fase aguda 234518213651 5255 , o acompanhamento por muitos anos parece indicar que entre 10 a 20 anos da doença manifestase mais freqüentemente a cardiopatia chagásica ${ }^{621}$ 2223272836 50. Podemos concluir a legitimidade de tal afirmativa ao observarmos (Tabela 6) em nosso material, uma tendência ao aumento gradativo das alterações no ECG na proporção de $2,52 \%$ ao ano.
Como já exposto anteriormente, evidências originadas de estudos de modelos animal e/ou humano sobre a fisiopatologia da CCC sugerem que esta doença ocorre como consequência de vários descritos e progressivo processo fisiopatológico ocorrendo após infecção. Coletivamente os eventos associados à infecção comprometem a micro vasculatura funcionalmente e resultam na hipoperfusão tendo como implicação uma fisiopatologia similar a outras cardiopatias crônicas congestivas. Como o eletrocardiograma é um dos meios de diagnóstico que permite evidenciar comprometimento miocárdio, em nosso material (Figura 3 e Tabela 6) sendo um estudo ao longo dos anos, nota-se uma progressiva incidência de anormalidades no eletrocardiograma principalmente bloqueio de ramo direito e hemibloqueio anterior esquerdo (Tabela 4).

$\mathrm{Na}$ fase crônica, as alterações da onda $\mathrm{T}$ podem assumir aspectos morfológicos variados, desde o inespecífico até o isquêmico, simulando doença coronariana. Constatamos a presença de anormalidade da onda $\mathrm{T}$ tipo isquêmica em apenas $7,4 \%$ de nosso material, explicado talvez pelo predomínio da faixa etária baixa de nossos casos (Figura 1). Uma das limitações de nosso trabalho foi a não realização de outros exames para afastar doença coronariana, justificada, talvez pelo caráter retrospectivo do mesmo.

Em trabalhos já publicados ${ }^{14}{ }^{15}$, não encontramos nenhuma modificação quanto às alterações eletrocardiográficas desses mesmos pacientes quando tratados na fase aguda. Do mesmo modo, Teixeira ${ }^{13} 56$, em trabalho experimental em coelhos, analisando o efeito da administração de benzonidazol na evolução da miocardite chagásica, conclue que as lesões histopatológicas residuais encontradas nos coelhos tratados foram qualitativamente similares àquelas vistas nos animais infectados com $T$. cruzi mas que não receberam tratamento específico.

\section{Limitação de nosso trabalho}

De alguma maneira, nosso material, não representa a história natural da doença de Chagas. Trata-se de casuística selecionada e de caráter descritivo com dados de freqüência de características 
Pedrosa RC, Cançado JR, Decache W. Estudo longitudinal do eletrocardiograma na doença de Chagas desde a fase aguda. Revista da Sociedade Brasileira de Medicina Tropical 26:163-174, jul-set, 1993.

Tabela 7 - Dados radiológicos: o indice cardiotorácico de trinta e dois casos.

\begin{tabular}{lcccccc}
\hline & \multicolumn{7}{c}{ Grupos etários } \\
\cline { 2 - 7 } & $0-10$ & $11-20$ & $21-30$ & $31-40$ & $41-50$ & $51-60$ \\
\hline$<50$ & 3 & 7 & 2 & 2 & 2 & 1 \\
$\geqslant 50$ & 10 & 2 & 1 & - & 1 & 1 \\
\hline
\end{tabular}

ICT $=$ indice cardiotorácico

clínicas. Entretanto, tais fatos não invalidam nossas observações uma vez que verificamos que não houve modificações evolutivas eletrocardiográficas na dependência do tratamento e os pacientes retornaram ao local de origem. Por outro lado, não julgamos no direito de compararmos a um estudo longitudinal realizado em população não selecionada que a nosso ver é o modelo adequado à observação de um doença.

\section{Conclusões}

a) Os autores concluem que as alterações eletrocardiográficas agravam com a evolução da doença sendo que após a fase aguda pode ocorrer regressão das mesmas. Digno de nota foi a presença de hemibloqueio posterior esquerdo na fase aguda. b) $O$ eletrocardiograma não serve como índice de avaliação terapêutica.

\section{SUMMARY}

Several studies have been done to analyse the relationship between the characteristics of the electrocardiogram (ECG) and mortality in the several stages of the disease, using different methods like multiple case and longitudinal studies. We analysed the ECG from the acute stage up to twenty years of follow-up $1 \pm 9$ years) in 42 patients with Chagas' disease to determine their evolution and it's value like an index for therapeutic evaluation. The 42 patients (18 female, 24 males) were originally from the North of the State of Minas Gerais (Brazil) and the initial stage was mainty in the first two decades of age. All bad cardiac involvement and received full specific treatment. We utilized the following criteria for the ECG analyses: Modified Minnerota Code for Chagas' disease; WHO/ISFC TASK FORCE for inter ventricular conduction disturbances and Pieretti criteria for inactive electrical areas. We conclude that: a) The electrocardiogram changes tend to get worse with evolution into the chronic stage; $b$ ) The electrocardiogram is not a good index for therapeutic valuation.

Key-words: Chronic chagasic cardiopathy. Longitudinal study. Resting eletrocardiography.

\section{REFERÊNCIAS BIBLIOGRÁFICAS}

1. Amorim DS, Manço JC, Godoy RA, Gallo Jr L. Clínica-forma crônica cardíaca. In: Brener ZA, Andrade $Z$ (eds) Trypanosoma cruzi e doença de Chagas. Editora Guanabara Koogan, Rio de Janeiro, p.265, 1979.

2. Andrade SG. Aspectos da patologia da doença de Chagas. In: Anais do Simpósio sobre Moléstia de Chagas. São Paulo p.72, 1979.

3. Andrade ZA. Patologia do sistema excito-condutor do coração na miocardiopatia chagásica. Revista de Patologia Tropical 3:367, 1974.

4. Andrade ZA, Andrade SG, Sadigursky M, Costa Lima JA. Doença de Chagas Experimental no cão. Relação morfológica e eletrocardiográfica na fase aguda da in fecção. Arquivo Brasileiro de Cardiologia $35: 485,1980$.

5. Anselmi A, Pifano FC, Suarez MD, Domingues MD. Experimental Schizotrypanum cruzi Myocarditis. Correlation between histopathologic and eletrocardiographic finding in experimental Chagas' heart disease. American Heart Journal 70:638, 1965 .

6. Barros LC. O eletrocardiograma na forma crônica da moléstia de Chagas. O Hospital 35:705, 1949.

7. Benchimol A. Características clínicas de 75 casos de cardiopatia chagásica observados no Rio de Janeiro. In: Resumos do Congresso Interamericano de Cardiologia, Rio de Janeiro, 1960.

8. Brasil A. A variabilidade eletrocardiográfica na cardiopatia crônica chagásica. Revista da Associação Médica de Minas Gerais 4:149, 1953.

9. Brasil A. Evolução prognóstico da doença de Chagas. Arquivo Brasileiro de Cardiologia 18:365, 1965. 
10. Brasil A. Aforismos acerca da "Doença de Chagas" de rítmos cardíacos e esofagianos. In: Modernos Conhecimentos sobre a Doença de Chagas. Universidade Federal de Minas Gerais/Academia Mineira de Medicina, Belo Horizonte p.50, 1981.

11. Brener Z, Andrade Z. Trypanosoma cruzi e doença de Chagas. Guanabara Koogan, Rio de Janeiro, 1979.

12. Caieiro T, Palmero HA. Estúdio de la sobrevida de una problacion con enfermedad de Chagas crônica. Medicina (Buenos Aires) 42(Supl. 1):15-21, 1982.

13. Cançado JR, Chuster M. Cardiopatia Chagásica. Fundação Carlos Chagas, Belo Horizonte, 1985.

14. Cançado JR, Marra UD, Mourão OG. Bases para avaliação do tratamento específico da doença de Chagas humana. Revista da Sociedade Brasileira de Medicina Tropical 7:155, 1973.

15. Cançado JR, Pedrosa RC. Aspectos evolutivos da Cardiopatia Aguda. In: Cançado JR, Chuster M (eds) Cardiopatia Chagásica. Fundação Carlos Chagas, Belo Horizonte, cap.15, 1985.

16. Carrasco MA, Guerrero L, Henry PW, Cesar M, RafaelC. Ventricular arrhythmias and left ventricular myocardial function in chronic chagasic patients. International Journal Cardiologic 28:35-41, 1990.

17. Chagas C. A forma cardíaca de tripanosomiase americana. Archivo Brasileiro de Medicina 18:46, 1928.

18. Chapadeiro E, Tafuri WL, Lopes ER. Anatomia Patológica da Cardiopatia Aguda. In: Cançado JR, Chuster M (eds) Cardiopatia Chagásica. Fundação Carlos Chagas, Belo Horizonte, cap. 6, 1985.

19. Coura JR, Pereira JB, Henry PW. Morbidade da Doença Chagas IV. Estudo longitudinal de dez anos em Paim e Iguateme. Memórias do Instituto Oswaldo Cruz 80:73-80, 1985.

20. De Paola AAV. Estimulação Ventricular programada em pacientes com Cardiopatia Chagásica Crônica e Taquicardia Ventricular. Tese de Livre Docência. Escola Paulista de Medicina, São Paulo, 1991.

21. Dias JCP. Doença de Chagas em Bambuí, MG, Brasil. Estudo clínico-epidemiológico a partir da fase aguda entre 1940 e 1982. Tese de Doutorado, Faculdade de Medicina da Universidade Federal de Minas Gerais, Belo Horizonte, 1982.

22. Dias JCP. História natural. In: Cançado JR, Chuster M (eds) Cardiopatia Chagásica. Fundação Carlos Chagas, Belo Horizonte, 1985.

23. Dias E, Laranja FS. Estudo preliminar de inquéritos sorológicos - eletrocardiográficos em população não selecionadas de zonas não endêmicas e de zonas endêmicas de doença de Chagas. Revista Brasileira de Malariologia e Doenças Tropicais 5:205, 1953.

24. Factor SM, Cho S, Tanowitz HB. Anormalities of the coronary microcirculation in acute Murine Chagas' disease. American Journal Tropical Medicine 34:246-253, 1985.

25. Foreign-born population by place of birth-selected characteristics: 1980. In: Bureau of the Census. Statistical abstract of the United States, $110^{\text {th }}$ edition. Government Printing Office, Washington D.C. p.41, 1990.

26. Forichon E. Contribution aux estimations de morbideté et de mortalité dans la Maladie de Chagas. Revista de Patologia Tropical 4:57-78, 1975.

27. Gruppi A, Gea S. Human antibodies against Trypanosoma cruzi exo antigens recognizing parasite surface antigens and heart tissue components. International Archuives Allergy Application. Immunologic 90:119-232, 1989.

28. Guimarães AC. Quadro clínico da miocardite crônica chagásica. Revista de Medicina da Bahia 25:238$243,1979$.

29. Guimarães AC. Aspectos eletrocardiográficos de cardiopatia crônica. In: Cançado JR, Chuster M (eds) Cardiopatia Chagásica. Fundação Carlos Chagas, Belo Horizonte, 1985.

30. Higuchi ML, De Morais CF, Lopes EA, Saldanha LB, Barreto ACP. Histopathological criteria of myocarditis; study based on normal heart, chagasic heart and dilated cardiomyopathy. Japonese Circulation Journal 54:391 400, 1990.

31. James MH, Rahmitoola SH. Chagas' disease heart in the United States. New England Journal Medicine 325:763-768, 1991.

32. Kirchoff LV, Gam AA, Neva FA. American trypanosomiases (Chagas' disease) in Central American immigrants. American Journal Medicine 82:915, 1987.

33. Köberle F. Patologia y anatomia patológica de la enfermidad de Chagas. Bolletin Oficial Sanitarismo Panamericano 51:404-428, 1961

34. Laguens RP, Merckert PC. Anti heart antibody dependent by to toxicity in the sera of mice chronically infected with Trypanosoma cruzi infectologic. Immunologic 56:993-987, 1988.

35. Laranja FS, Dias E. Chagas' disease. A clinical, epidemiologic and pathologic study. Circulation 14:1035, 1956.

36. Laranja FS, Dias E, Nóbrega G. O eletrocardiograma na Cardiopatia crônica da doença de Chagas. Brasil Médico 62:51, 1948.

37. Lopes ER, Chapadeiro E. Morte súbita em área endêmica da doença de Chagas. Revista da Sociedade Brasileira de Medicina Tropical 16:79-84, 1983.

38. Macedo VO. Influência da exposição à re-infecção na evolução da doença de Chagas. Tese de Mestrado, Universidade Federal da Bahia, Salvador, 1973. 
Pedrosa RC, Cançado JR, Decache W. Estudo longitudinal do eletrocardiograma na doença de Chagas desde a fase aguda. Revista da Sociedade Brasileira de Medicinq Tropical 26:163-174, jul-set, 1993.

39. Maguire JH, Holl R, Sherlock I, Guimarães AC, Sleigh A, Ramos NB, Mott KE. Cardiac morbidity and mortality due to Chagas' disease: prospective eletrocardiographic suty of a Brazilian Community. Circulation 75:1140-1445, 1987.

40. Maguire JH, Mott KE, Souza JAA. Eletrocardiographic classification and abbreviatel len $d$ system for population based studies of Chagas' disease. Bulletim Pan American Health Organization 16:47, 1982.

41. Marsden PD. Selective primary health care: strategies for control of disease in the developing world XVI Chagas disease. Reviews of Infections Diseases 6:855-865, 1984.

42. McCormick TS, Rowland EC. Trypanosoma cruzi: cross-reactive anti-heart anti-antibodies produced during infection in mice. Experimental Parasitologic 69:393-401, 1989.

43. Milei J, Mautuer B, Storino R, Julian AS, Victor JF. Does Chagas disease exist as an undiagnosed form of cardiomyopathy in the United States. American Heart Journal 123:1732-1735, 1992.

44. Moreira CHS. Contribuição ao estudo da forma crônica assintomática da doença de Chagas. Tese de Mestrado, Universidade Federal da Bahia, Salvador, 1975.

45. Morris SA, Tanowitz HB, Wittner M, John PB. Pathophysiological Insights into the cardiomyopathy of Chagas' Disease. Circulation 82:1900-1909, 1990.

46. Mota ELA. Mortalidade em uma população de área endêmica para a doença de Chagas. Um estudo longitudinal de 9 anos em Castro Alves. Tese de Doutorado, Universidade Federal da Bahia, Salvador, 1988.

47. Pan American Health Organization. Health condictions in the Americas. Scientific publication 524 Vol. 1 Washington, D.C., Pan American Health
Organization:160-164, 1990.

48. Pieretti $\mathrm{OH}$. Diagnostic eletrocardiográfico da zona inativa da miocardite crônica. Revista de la Sociedad Venezuelana de Cardiologia 1:73, 1958.

49. Porto CC. Contribuição do eletrocardiograma no prognóstico e evolução da doença de Chagas. Tese de Doutorado. Universidade Federal de Minas Gerais, Belo Horizonte, 1963.

50. Pugliese C, Lessa I. Estudo da sobrevida na miocardite crônica de Chagas descompensada. Revista do Instituto de Medicina Tropical de São Paulo 18:191-201, 1976.

51. Rassi A, Carneiro O. Estudo clínico eletrocardiográfico e radiológico da cardiopatia chagásica crônica. Análise de 106 casos. Revista Goiana de Medicina 2:289, 1956.

52. Rassi A. Clínica: fase aguda. In: Brener $Z$, Andrade $\mathbf{Z}$ (eds) Trypanosoma cruzi e doença de Chagas. Guanabara Koogan, Rio de Janeiro, 1979.

53. Rossi MA. Microvascular Changes as a cause of chronic cardiomyophaty in Chagas' disease. American Heart Journal 120:233-236, 1960.

54. Sadigursky M, Von Kreuter BF. Association of elevated anti-sarcolema anti idiotype antibody levels with clinical and pathologic expression of chronic Chagas myocarditis. Circulation 80:1269-1276, 1989.

55. Tafuri WL. Patogenese, In: Cançado JR, Chuster M (eds) Cardiopatia chagásica. Fundação Carlos Chagas, Belo Horizonte, cap. 1, 1985.

56. Teixeira ARL, Solórzano E, Zappala M, Rizzo LV. Efeito da administração de benzonidazol na evolução da miocardite chagásica experimental. In: Resumos da Reunião Anual sobre Pesquisa Aplicada em Doença de Chagas. Araxá, Fundação Carlos Chagas, Belo Horizonte p.32, 1985. 